\title{
Kikuchi Necrotising Lymphadenitis in a Child: A Rare Case Report
}

\author{
Sachin Goel Ankur Gupta Kanwar Sen Pooja Swami \\ Department of Otorhinolaryngology and Head \& Neck Surgery, ABVIMS \& RML Hospital, Baba Kharak \\ Singh Marg, Delhi, India
}

\section{Keywords}

Kikuchi-Fujimoto disease · Lymphadenopathy · Necrotising lymphadenitis

\begin{abstract}
Introduction: Kikuchi-Fujimoto disease (KFD) is a rare benign disease with a self-limiting course. Patients usually present with clinical features of tender cervical lymphadenopathy (LAP), fever, malaise, weight loss, and night sweats. The disease may mimic infective LAP or lymphoma on clinical and histopathological examination (HPE). Case Report: We report a case of 3-year-old male child who presented to us with bilateral cervical LAP not responding to conservative management. Excision biopsy was done and HPE revealed KFD. Conclusion: Clinicians should have high index of suspicion for diagnosis of KFD in patients with cervical LAP not responding to conservative management. Excision biopsy is important for accurate diagnosis and to avoid aggressive interventions like chemotherapy. ( 2020 The Author(s)

Published by S. Karger AG, Basel
\end{abstract}

\section{Introduction}

Kikuchi-Fujimoto disease (KFD) or Kikuchi necrotizing lymphadenitis was first described by Kikuchi and Fujimoto in 1972 in Japan [1]. It is a type of histiocytic necrotizing lymphadenitis which is benign and self-limiting.
It is predominantly seen in adult females of Asian origin and is rarely seen in Western countries. It is characterized by tender cervical lymphadenopathy (LAP) and flu-like symptoms. The etiopathogenesis of KFD is still not clear, but various infectious agents like Toxoplasma gondii, Yersenia enterocolitica, human herpesvirus $6 \& 8$, hepatitis-B, and Epstein-Barr virus have been implicated [2-4]. The role of immunological mechanisms has also been proposed for the pathogenesis of KFD. The patients can be misdiagnosed as either systemic lupus erythematosus or lymphoma, and it becomes imperative to diagnose KFD to avoid unnecessary interventions. We report a rare case of KFD in a male child.

\section{Case Report}

A 3-year-old male child presented to ENT Department of Atal Bihari Vajpayee institute of medical sciences and Dr Ram Manohar Lohia Hospital (ABVIMS \& Dr RMLH), New Delhi, with swelling over the right side of the neck for the past 3 days and fever and lethargy for the past 1 day. He had a history of night sweats and excessive crying. Patient was febrile with no signs of dehydration. Examination revealed diffuse inflammatory swelling of the right side of neck extending from just below the angle of mandible to the level of hyoid bone. Swelling was tender but non-fluctuant.

Ultrasonography (USG) neck revealed bilateral multiple nonnecrotic cervical LAP involving level $1 \mathrm{~b}, 2,3,4$ and 5, with conglomeration but maintained fatty hilum. Largest lymph node was seen at level 2 which measured $18 \mathrm{~mm}$ in maximum diameter. There was no suppuration/collection on USG neck. USG abdomen was normal.

$\begin{array}{ll}\text { karger@karger.com } & \text { (c) 2020 The Author(s) } \\ \text { Published by S. Karger AG, Basel } & \text { Karger } \\ \text { Kargen ciccess } & \text { This article is licensed under the Creative Commons Attribution- } \\ \text { NonCommercial-NoDerivatives 4.0 International License (CC BY- } \\ \text { NC-ND) (http://www.karger.com/Services/OpenAccessLicense). } \\ \text { Usage and distribution for commercial purposes as well as any dis- } \\ \text { tribution of modified material requires written permission. }\end{array}$

Sachin Goel

Senior Resident, Department of Otorhinolaryngology and Head \& Neck Surgery ABVIMS \& RML Hospital

Baba Kharak Singh Marg, New Delhi 110001 (India)

sachin13jan@gmail.com 
Table 1. Showing the results of serial CBC

\begin{tabular}{llllll}
\hline Parameter & Day 1 & Day 2 & Day 4 & Day 7 & Day 11 \\
\hline ESR, mm 1st h & 77 & 65 & 52 & 33 & 30 \\
Hb, g & 6.5 & 7 & 10 & 10.5 & 11.2 \\
TLC, per mm ${ }^{3}$ & 38,000 & 34,000 & 16,600 & 17,600 & 14,000 \\
Polymorphs & 85 & 84 & 65 & 49 & 47 \\
Lymphocyte & 12 & 13 & 26 & 48 & 51 \\
Eosinophil & 1 & 2 & 4 & 1 & 1 \\
Monocyte & 1 & 1 & 5 & 2 & 1 \\
Basophil & 0 & 0 & 0 & 0 & 0 \\
Red blood cells & 5.74 & 5.0 & 5.6 & 5.98 & 5.5 \\
Mean corpuscular volume & 55 & 31 & 60 & 68 & 62 \\
Mean corpuscular hemoglobin concentration & 28 & 31.9 & 30.4 & 28 & 28.6 \\
\hline
\end{tabular}

CBC, complete blood count.

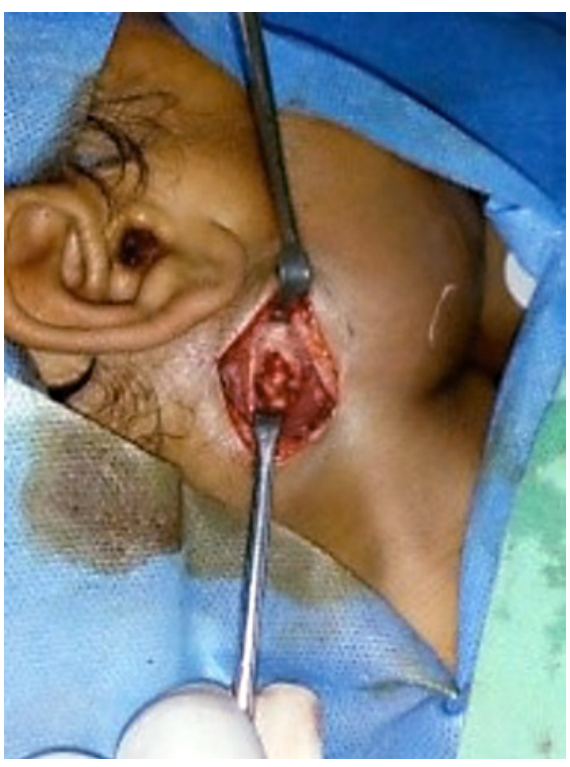

Fig. 1. Lymph node excision biopsy under GA. GA, general anesthesia.

The patient was severely anemic, and erythrocyte sedimentation rate (ESR) was found to be increased on complete blood count (CBC). Leukocytosis with increased polymorphs was identified initially which progressed to increase in lymphocytes over time (shown in Table 1).

Mantoux test was negative and chest X-ray posteroanterior view was normal. All viral markers were found to be nonreactive, and routine urine examination was also normal. Lactate dehydrogenase level was increased.

Intravenous ceftriaxone was started half gram twice a day, and vancomycin was added later on after pediatrician advice. The patient showed no signs of improvement even after 4 days of conservative management, and therefore, lymph node excision biopsy was done under general anesthesia (shown in Fig. 1).

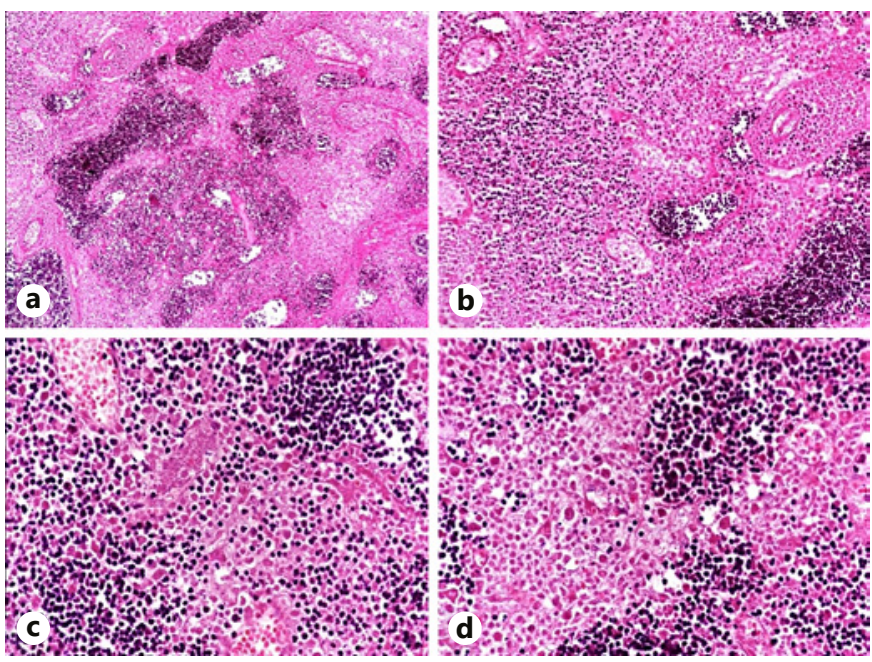

Fig. 2. a, b Multiple pale circumscribed foci of necrotic lesions, giving starry sky appearance. c, d Karyorrhectic debris, histiocytes, fibrin deposits, and plasmacytoid monocytes along with activated lymphoid cells.

No pathogen or tubercular granulomas were seen on histopathological examination (HPE). The same showed effaced architecture with large areas of paracortical necrosis with abundant karyorrhectic debris, scattered fibrin deposits, and mononuclear cells. Many plasmacytoid dendritic cells were seen. Ziehl-Neelsen (ZN) stain for acid fast was negative. No atypical cells were observed. Overall morphology was suggestive of Kikuchi necrotizing lymphadenitis (shown in Fig. 2a-d).

The patient was started on oral ibuprofen. The patient recovered completely with improvement in fever and pain after 4 weeks of treatment. Antinuclear antibody titers came out to be normal. Patient is under our regular follow-up for past 3 months, and there is no evidence of any complication or recurrence. 


\section{Discussion}

KFD is a benign, self-limiting rare cause of unilateral cervical LAP. The size of lymph node is usually $1-4 \mathrm{~cm}$, but cases up to $8 \mathrm{~cm}$ have been reported. Occasional cases show involvement of mediastinal, peritoneal, retroperitoneal, and inguinal regions. The patient often presents with low-grade fever, malaise, oral ulcers, joint pains, and hepatosplenomegaly. CBC usually shows mild leucopenia with increased ESR and C-reactive protein levels; however, in our case, the patient presented with leukocytosis. The serological tests, including autoimmune antibodies and urine examination, are usually normal.

KFD may be associated with Epstein-Barr virus, implanted pacemakers, meningitis, breast cancer, and buccal cancer $[2,5]$. The diagnosis is confirmed on HPE. KFD resolves spontaneously within a few months. According to available literature, KFD is a benign disease, but some proportion of people may develop complications like pulmonary hemorrhage, fatal hemophagocytic syndrome, and heart failure [6]. The treatment is essentially symptomatic with analgesics and antipyretics. Good results have also been reported with intravenous immune globulins in patients with severe symptoms; however, the role of antibiotics is controversial.

\section{Conclusion}

KFD is a rare benign cause of LAP. It should always be considered as a differential diagnosis in cases of LAP with generalized symptoms of malaise, fever, and lethargy. It may mimic tubercular LAP or malignant lymphoma, and HPE is essential for accurate diagnosis and to avoid aggressive treatment. An early diagnosis will prevent the patient from mental, financial, and emotional stress. Long-term follow-up is important as patients may develop systemic lupus erythematosus or lymphoproliferative malignant disease later in life.

\section{Statement of Ethics}

Written and informed consent was obtained from the patient's father to publish the case. Also, approval was obtained from the Institution Ethics Committee, ABVIMS \& RML Hospital, for reporting the case.

\section{Conflict of Interest Statement}

The authors have no conflicts of interest to declare.

\section{Funding Sources}

The authors did not receive any funding.

\section{Author Contributions}

S.G.: writing - original draft preparation and visualization. A.G.: writing - reviewing and editing. K.S.: supervision. P.S.: investigation.

\section{References}

1 Menasce LP, Banerjee SS, Edmondson D, Harris M. Histiocytic necrotizing lymphadenitis (Kikuchi-Fujimoto disease): continuing diagnostic difficulties. Histopathology. 1998 Sep;33(3):248-54.

2 Ifeacho S, Aung T, Akinsola M. Kikuchi-Fujimoto disease: a case report and review of the literature. Cases J. 2008;1(1):187-9.

3 Bowman JJ, Hee CQ. Kikuchi's lymphadenitis: cervical lymphadenopathy suggestive of acute lymphoma. ANZ J Surg. 2007;77(5): 403-4.
4 Hudnall SD, Chen T, Amr S, Young KH, Henry K. Detection of human herpesvirus DNA in Kikuchi-Fujimoto disease and reactive lymphoid hyperplasia. Int J Clin Exp Pathol. 2008;1(4):362-8.

5 Quadri F, Atkin GK, Das SK. Kikuchi's disease: an important cause of cervical lymphadenopathy. Clin Med. 2007;7(1):82-4.

6 Perry AM, Choi SM. Kikuchi-Fujimoto disease: a review. Arch Pathol Lab Med. 2018 Nov;142(11):1341-6. 